\title{
Environmental Software Systems In Water Resources: Problems and Approaches Workshop Report
}

\author{
Robert M. Argent \\ Centre for Environmental Applied Hydrology, The University of Melbourne, Parkville, 3052, \\ Australia,R.Argent@civag.unimelb.edu.au
}

Key words: Water Resource Information Systems

Abstract: Problems exist in the development and use of environmental software systems in water resources. These include a legacy of feature-poor, inflexible, problem-specific software, and a lack of modern software engineering (SE) practice in recent or new system development. A workshop on the common problems of environmental software systems in water resources was held, and participants considered issues of user involvement, data handling, component design, and a framework for model selection, design, development and execution. The workshop outcomes highlighted the availability of a range of modern SE tools, techniques and processes that can be adopted to solve many of the problems that exist. Workshop participants were W.J. Young, A. Sokolov, M. Olshammar, C. Maul, L.F. Leon, V. Keskisarja, A. Jolma, T.F.N. Farley, S. Dorner, K. Brown, and R.M. Argent (convenor).

\section{INTRODUCTION}

With water resources use increasing across the globe, and issues arising in areas as diverse as water supply and wastewater disposal, flood forecasting and planning, water quality, and freshwater habitat, there is a corresponding need for sound water resources management with appropriate use of computer tools. Use of environmental models and software systems in water resources has a long history, with water resources engineers and scientists being early adopters of computer technology to undertake planning and management calculations that, previously, were done manually. Some of the earliest environmental information systems were developed to meet the needs of water resources managers, and such systems are continually 
being developed to meet new demands in data collection, storage, analysis and application.

Today, software systems are used in research, education, planning and management of water resources (McDonald and McAleer, 1997; El-Swaify and Yakowitz, 1998). These systems range widely in design and application, and include process models, such as MIKE-SHE (Refsgaard and Storm, 1995), conceptual models (e.g. Huber, 1995), simple correlation relationships, system analysis models (e.g. Ivanov et al., 1996), and operational models (e.g. Henry et al., 1988).

As is generally the case, early adoption of computer technology and modelling approaches has resulted in some drawbacks, particularly when the features of existing models are compared to modern software engineering (SE) concepts. Often, existing models were developed before establishment of modern SE principles, and were designed for specific purposes or sites by research scientists and engineers not well schooled in SE. Many models have been designed and built as "monoliths", consisting of large blocks of (hopefully, structured) code. This makes re-use of models difficult, except in situations similar to those for which the models were originally designed. Linking and extension of existing monolithic models is even more difficult, even when they are being applied as their makers intended. In constructing models for individual use, developers have used approaches, shortcuts or assumptions that are only appropriate to the task at hand, and which go further to making models inaccessible to all but those with sufficiently high technical skills and understanding of the phenomena being modelled. This, of course, has the advantage that misuse of the model is less likely, as users are required to be experts, but it does limit utilisation of a large effort in design and coding to a small range of people and applications.

Another legacy of monolithic models that has been observed, for example in surface and sub-surface hydrological models, is the plethora of input and output approaches. Many of these models have similar input requirements, such as climate and topographic data, but have a multitude of input file formats and loading styles. Similarly, model output is treated in a range of ways, from fully integrated maps, graphs and tables, to text files that then have to be loaded and manipulated in separate spreadsheet, GIS or word processing tools to reach the final end-point of an output data presentation.

Software engineering theory, techniques and tools offer a variety of solutions to the problems outlined above, from model description and design to protocols for software linking and embedding. At the $3^{\text {rd }}$ International Symposium on Environmental Software Systems, a workshop was held to discuss possible approaches to increasing utilisation of SE and other approaches to improve our design, development and use of environmental 
software systems in water resources. This paper reports the content and findings of that workshop.

\section{WORKSHOP PREPARATION AND PROBLEM SPECIFICATION}

Prior to the workshop, conference delegates were contacted via email to establish areas of interest that may have been suitable for workshop topics. Issues that were canvassed included:

- the broad range of software systems used, and the lack of a common software system language for use in water resources;

- the broad range of physical and management environments within which software is used;

- the merits of purpose building software for each new application, as opposed to use of existing software, along with consideration of the issues involved in deciding on components and the level of integration to build into software;

- the identification and inclusion of user needs in software development, including the problem of attracting user criticism of underdeveloped software by involving potential users directly in development;

- development and incorporation of metadata, benchmarks, and compliance testing in our software, and

- building redundancy and software timecycle concepts into our software design.

After discussion between symposium delegates, two topics were developed for initial consideration by the workshop, as follow:

1. Application of sound SE principles within water resources software development.

Many models used in water resources were developed decades ago, before modern SE principles were established. In an environment where these models are widely used, and new models often developed by researchers with little SE training, how do we encourage the adoption of sound software design? Do we agree on the principles of sound software design? - e.g. platform independence, alternative and complementary technologies, object-oriented design, metadata incorporation, benchmarks, peer review, model validation, built-in-redundancy, software timecycles? Would the establishment of protocols for water resources software that incorporate these principles be one solution? Should we just build a suite of feature-rich applications/libraries that handle all our data input needs and output interpretation, and leave it to individual researchers to furnish the 
model algorithms? Are we worrying about a problem that will disappear as new models, particularly spatially explicit modelling, are developed?

2. The identification and inclusion of user needs in software development.

A fundamental principle of software design in water resources is user involvement. How do we go about allowing users to have a significant input to software development? Are there some fundamental principles of user involvement, such as specific points of user input in design, or offering distinct alternatives from which users must choose a preferred approach? How do we avoid the problem of ill-informed users losing faith in the model when they have examined an underdeveloped prototype?

These topics were distributed to symposium delegates for consideration at the water resources workshop. The workshop was subsequently attended by eleven of the conference delegates, from four countries.

\section{WORKSHOP DISCUSSION}

The workshop started with some discussion of the background and the experiences of the delegates. This was followed with a presentation, by the workshop convenor, of a conceptual or meta-model framework, with input from delegates on framework components, and problems to be dealt either within or without the framework. Four specific areas of concern for the workshop attendees came out of this discussion, and were selected for further small-group discussion. The four specific problems were defined as:

- user needs and involvement;

- data handling;

- component specification, design and object-oriented design issues, and

- modelling framework.

These four areas formed the basis of discussion for the remainder of the workshop, and form the basis of the workshop findings.

\section{SMALL GROUP DISCUSSION}

The small groups met for a few hours to discuss these problems more fully, and were asked to provide a clear definition of the problem, some examples of the problem and solutions, and any principles or strategies that were pertinent to the problem solution. 


\subsection{User needs and involvement}

The problem of user needs and involvement in software design and development were defined clearly in the second question posed to workshop attendees, and, in essence, revolve around the processes required to adequately and appropriately involve users in the design and development of environmental software systems. The reasons for this are that there are many types of users of a given software tool, with a variety of needs, skills and experience. Use of a model or system will increase if, during development, users have the opportunity to both influence the design to meet their needs, and to become familiar with the software and its limitations and assumptions (Loucks and da Costa, 1991; Sprague and Carlson, 1982; Guariso and Werthner, 1989). Examples of not meeting user needs can be found in much of the software that is sitting on the readers shelves, unused (or unopened) because it failed to meet the needs of the users.

Approaches to overcome this problem include, firstly, the identification of users. The potential users of a system can be much broader than originally expected, because environmental software systems can be used in a number of different ways. For example, a decision support system that holds data such as spatial distribution of land use and population, may be used simply as a data source for another project, as well as in a decision support role. Following on from the identification of users is the establishment of users groups, so that like-use users can combine their interests and expertise to form development input.

Periodic consultation with users duririg development helps, as users can give feedback on software design, and also become familiar with the software layout and operation. The availability of computer aided software engineering (CASE) tools with rapid application facilities aids this process, and allows rapid prototyping of interfaces (Loucks et al., 1985), thereby enhancing the capacity for users to influence software design to meet their needs. One more strategy for incorporating user needs within software development is to establish user categories and profiles, to enhance identification of only those users with interests relevant to the current software development.

\subsection{Data handling}

Data handling was defined as the activities that took place between data collection and use of data in a model, a software system, or in interaction with the user. Problems in data handling include data generation, merging data sets, knowledge "mining", classification, reduction of dimensionality, data access, querying, obtaining and using proprietary data, and 
interpolation, gap filling and extrapolation. Furthermore, data handling includes associated metadata issues such as creating metadata, organizing data, formats, uncertainties, and integrity and validation. Table 1 lists examples of the approaches that were suggested for solving some of these problems.

Metadata issues should be handled by improved use of metadata, including consistent description of fields, standardized field names, and the adoption and use of existing standards (e.g. ANZLIC Core Metadata Elements for land and geographic directories in Australia and New Zealand).

The appropriateness of any of the approaches suggested in Table 1 needs to be determined in respect to the type, character, and use being made, of the data. For most of the problems listed there is more than one way to approach and solve the problem. Keeping data handling as simple and straightforward as possible is a useful principle, although this, naturally, also depends on data type and use. In determining approaches to data management it is therefore suggested that understanding user needs and requirements, and, hence, the ways in which data will be used, should be considered fundamental. Data handling across projects, systems and countries will be enhanced by the use and promotion of appropriate standards.

Table 1. Problems and possible solutions in data handling

\begin{tabular}{|l|l|}
\hline \multicolumn{1}{|c|}{ Problem } & \multicolumn{1}{c|}{ Possible Solution } \\
\hline Data generation & Models \\
\hline Merging datasets & Unknown - a possible research area \\
\hline $\begin{array}{l}\text { Classification, reduction of } \\
\text { dimensionality, and knowledge } \\
\text { "mining" }\end{array}$ & $\begin{array}{l}\text { Data mining (genetic algorithms, self organizing } \\
\text { maps, neural networks); statistical methods }\end{array}$ \\
\hline Accessing the data & $\begin{array}{l}\text { Database integrator; integration architecture } \\
\text { (CORBA) }\end{array}$ \\
\hline Querying data & Query language (SQL) \\
\hline Proprietary data & Purchase; reverse engineer \\
\hline $\begin{array}{l}\text { Interpolation, gap filling and } \\
\text { extrapolation }\end{array}$ & Kriging, splines, models \\
\hline Integrity and validation of data & Visualization, comparisons, reference sets \\
\hline Uncertainties associated with data & Bayesian approaches \\
\hline Different data formats & $\begin{array}{l}\text { Common language for report generation and data } \\
\text { collection (PERL, and use of Regular Expressions) }\end{array}$ \\
\hline $\begin{array}{l}\text { Organizing data and creating } \\
\text { metadata }\end{array}$ & $\begin{array}{l}\text { Database management systems, markup language } \\
\text { (XML) }\end{array}$ \\
\hline
\end{tabular}




\subsection{Component specification, design and object-oriented design issues}

The use of components or modules within water resources software systems has been increasing, with some small moves away from construction of complete monolithic models. There are, however, still many problems to be faced in the design, development and use of components, including testing, documentation, inconsistent interfaces, single-platform execution, data handling and input/output, and metadata issues such as support of metadata and data naming consistency. Examples of these problems can be found in some of the standalone DOS-based water quality models that use text input and output files, with little or no inclusion of metadata. There also exists a legacy of mainframe models, with output in report format, that are incompatible with desktop systems.

Solutions to the problems of data handling and metadata have been covered previously. The problems of single platform support can be tackled by program replacement or recompilation, and eventually by rewriting in a language and format that operates across different platforms. Wrappers and shells also have a role to play, along with packaging modules into dynamic link libraries (DLL) and other object based approaches. Documentation of modules and metadata is an important aspect of SE, and should, ideally, be done to agreed standards. Sufficiently detailed documentation will also enhance the capacity to compare model performance and output. The adoption of object-oriented design of modules is suggested, and adoption of a standard for model or module description, such as the Unified Modeling Language (UML) (Booch et al., 1999), will go further to enhance both design and description.

The adoption of these sound SE principles may be aided by close cooperation between software engineers and water resources scientists and engineers, possibly to the point where specialists, such as database designers, undertake development of different components.

Fundamental principles that should be adopted in component design are to design before programming, including specification of clear goals for the component, follow common design principles for user interfaces, limit the scope of a component, and avoid "tacking-on" features that should be in a different component. As with data handling, there may be multiple ways to achieve the same result, so, overall, be open to alternative approaches.

\subsection{Modelling framework}

The modelling framework problem arose out of considerations of monolithic models and inflexible software, similar to the problems examined 
by the other groups, but at a more conceptual, or "big picture", level. In essence the problem was defined as the absence of a framework (either physical or conceptual) within which all aspects of water resources modelling could be undertaken. Figure 1 shows the ideas and components identified for inclusion in a possible framework.

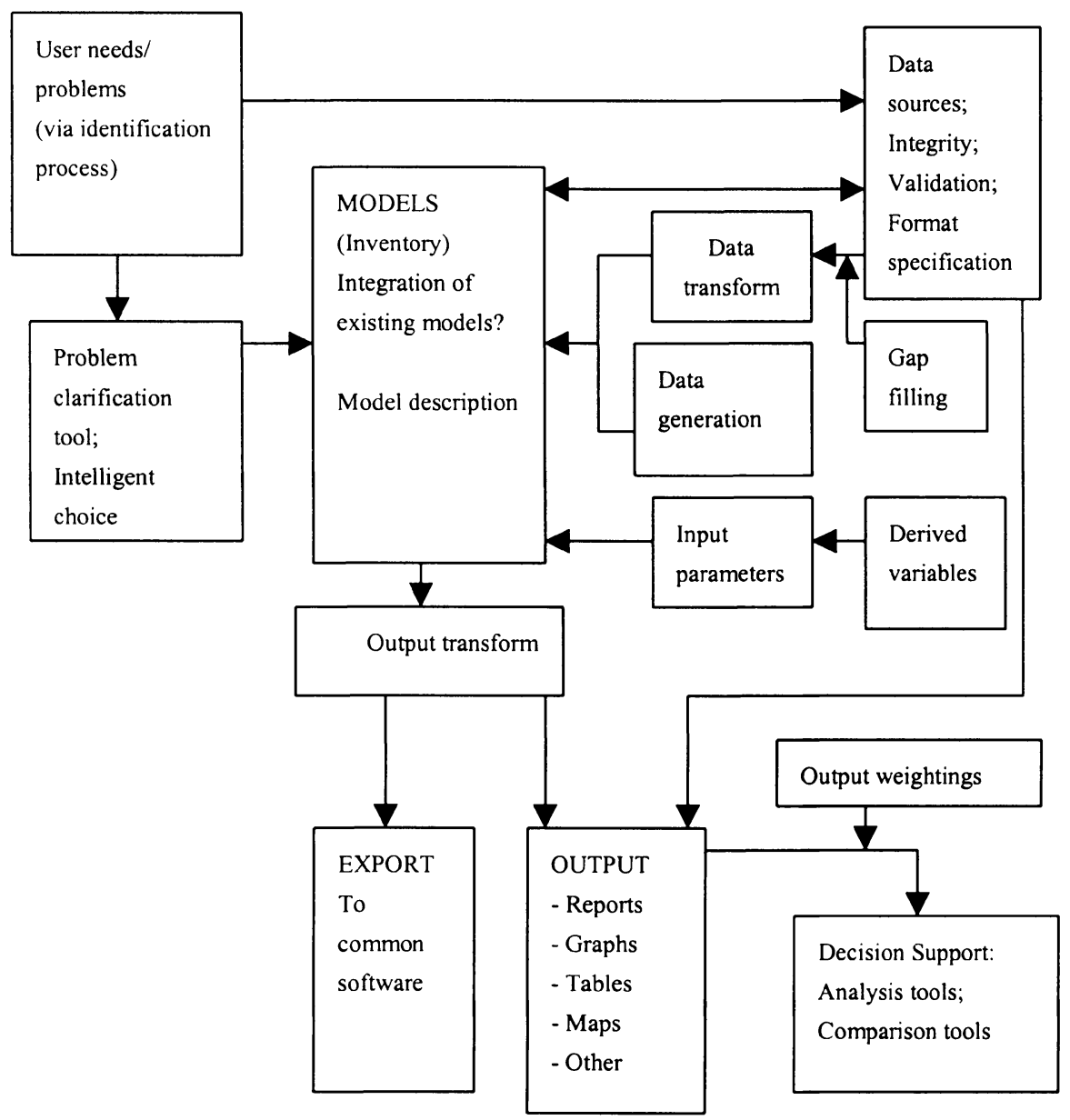

Figure 1. Water resources software framework

Desirable framework aspects included support for model development, data provision and checking, model selection and execution, output presentation and interpretation, and decision support. Features of the framework would include the ability to combine models, select data from different sources, options for output presentation, platform independence, open source component code and integrated documentation, covering use, 
requirements, assumptions and limitations, testing, validation, and external information sources.

Some examples of solutions or partial solutions can be found in various available integrated modelling approaches, such as the RAISON software (Lam, 1997; Leon et al., 1997) or GIBSI approach (Rousseau et al., 1997). Further enhancements to framework approaches could be made with adoption of modern SE tools, techniques and protocols, including visual object programming languages, scripting, communication and data protocols, markup languages, and documentation standards.

The framework shown in Figure 1 was developed by the attendees at the workshop, and is proposed as a conceptual approach that aims to combine not only the framework concepts but also the capacity to build in some of the ideas and suggestions of the other groups. External to the proposed framework are further issues such as overall architecture, documentation, and the needs for peer review, validation, testing, sensitivity analysis, and description of uncertainty in model outputs. Issues of redundancy and life cycles of software should also be included in overall design.

\section{DISCUSSION}

The outcomes of the workshop provided a range of solutions to the common problems of environmental software systems development and use in water resources. These solutions came not only from a range of new and emerging technologies, tools and techniques, but also from further adoption and utilisation of well known principles of software development. Despite our increasing understanding of the problems, and the solutions provided here, a problem still remains - how do we get non-SE trained engineers and scientists working in software development, principally in research, to adopt these types of approaches, and how do we stop them passing their bad habits on to proteges and students? Our approach should be to lead by example, by learning, adopting and displaying sound SE practices appropriate to the problems we are solving in water resources.

\section{ACKNOWLEDGEMENTS}

The input of the workshop participants and the support of conference sponsors is gratefully acknowledged. This work was supported by the Land and Water Resources Research and Development Corporation through project UME29. 


\section{REFERENCES}

Booch, G., Rumbaugh, J. and Jacobson, I. (1999) The unified modeling language user guide, Addison-Wesley, Reading.

El-Swaify, S. A. and Yakowitz, D. S. (Eds.) (1998) Multiple objective decision making for land, water, and environmental management, CRC Press, Boca Raton.

Guariso, G. and Werthner, H. (1989) Environmental Decision Support Systems, Ellis Horwood Limited, Chichester.

Henry, H. R., Hains, D. K., Burkett, E. and Schoel, W., III (1988) The Alabama rainfallrunoff model, ARRM In 3rd Water Resources Operations Management Workshop (Eds. Labadie, J. W., Brazil, L. E., Corbu, I. and Johnson, L. E.) Water Resources Planning and Management Division, American Society of Civil Engineers, Colorado State University, Fort Collins, Colorado, pp. 195-209.

Huber, W. C. (1995) EPA Storm Water Management Model - SWMM In Computer Models of Watershed Hydrology, Vol. 1 (Ed. Singh, V. P.) Water Resources Publications, Colorado, pp. 783-808.

Ivanov, P., Masliev, I., Kularathna, M., De Marchi, C. and Somlyody, L. (1996) Decision Support System for Evaluating River Basin Strategies. DESERT: Users Manual International Institute for Applied Systems Analysis, Laxenburg.

Lam, D. (1997) Decision support systems for water resource problems In MODSIM 97, Vol. 4 (Eds. McDonald, D. A. and McAleer, M.) Modelling and Simulation Society of Australia and International Association for Mathematics and Computers in Simulation, Hobart, pp. 1827-1834.

Leon, L. F., Lam, D. C., Swayne, D. A., Farquhar, G. J. and Soulis, E. D. (1997) Integration of a Nonpoint Source Pollution Model with a Decision Support System In Environmental Software Systems, Vol. 2 (Eds. Denzer, R., Swayne, D. A. and Schimak, G.) Chapman \& Hall, pp. 102-109.

Loucks, D. P. and da Costa, J. R. (Eds.) (1991) Decision Support Systems. Water Resources Planning, Springer-Verlag, Heidelberg.

Loucks, D. P., Kindler, J. and Fedra, K. (1985) Interactive water resources modeling and model use: an overview. Water Resources Research, 21, 95-102.

McDonald, A. D. and McAleer, M. (1997) Proceedings. MODSIM 97. International Congress on Modelling and Simulation, The Modelling and Simulation Society of Australia, Hobart, Australia.

Refsgaard, J. C. and Storm, B. (1995) MIKE SHE In Computer Models of Watershed Hydrology, Vol. 1 (Ed. Singh, V. P.) Water Resources Publications, Colorado, pp. 809846.

Rousseau, A. N., Maihot, A., Massicotte, S., Duchemin, M., Dupont, J. and Villeneuve, J. P. (1997) GIBSI: A watershed-based software system for the integrated management of surface water quality. Journal of Soil and Water Conservation, 25, 284-301.

Sprague Jr., R. H. and Carlson, E. D. (1982) Building Effective Decision Support Systems, Prentice-Hall, Englewood Cliffs, NJ. 